PROCEEDINGS OF THE

AMERICAN MATHEMATICAL SOCIETY

Volume 125, Number 8, August 1997, Pages 2221-2225

S 0002-9939(97)03908-7

\title{
ON GENERA OF SMOOTH CURVES IN HIGHER DIMENSIONAL VARIETIES
}

\author{
JUNGKAI ALFRED CHEN
}

(Communicated by Ron Donagi)

\begin{abstract}
We prove that for any smooth projective variety $X$ of dimension $\geq 3$, there exists an integer $g_{0}=g_{0}(X)$, such that for any integer $g \geq g_{0}$, there exists a smooth curve $C$ in $X$ with $g(C)=g$.
\end{abstract}

\section{INTRODUCTION}

It's a very elementary fact that, for any integer $g \geq 0$, there exists a smooth curve $C \subset \mathbb{P}^{3}$ of genus $g$. It is interesting to ask what the analogous situation is when projective space is replaced by an arbitrary smooth projective variety $X$ of dimension $\geq 3$. It can happen of course that a given variety $X$ contains no curves of small genus. For example, abelian varieties contain no rational curves, and Clemens [1] has shown that on a generic hypersurface of degree $d$ in $\mathbb{P}^{n}$, there are no curves of genus $\leq \frac{d-2 n+1}{2}$. So the natural question is whether all sufficiently large genera are realized by smooth curves. Our main result states that this is indeed the case.

Theorem 1. Let $X$ be a smooth projective variety of dimension $n \geq 3$. Then there exists an integer $g_{0}=g_{0}(X)$ such that for any integer $g \geq g_{0}$, there exists a smooth curve $C \subset X$ of genus $g$.

We also prove an analogous statement for the geometric genera of nodal curves on surfaces.

The paper is organized as follows. In section 1, we prove a lemma that a certain type of function will represent all large enough integers. In section 2, we construct a smooth surface $S$ in any smooth projective variety of $\operatorname{dim} \geq 3$ such that $\operatorname{Pic}(S)$ is very large. We choose divisors in $S$ which give us various genera. In section 3 , we prove the analogous statement for surfaces that any large enough integer can be realized as the geometric genus of some nodal curves on a given surface.

We work over the complex number $\mathbb{C}$.

We'd like to thank Robert Lazarsfeld for his encouragement and suggestion. We are indebted to Joe Harris and Claire Voisin for pointing out the importance of surfaces with large Picard groups. We would also thank Richard Elman, Mark Green and Christopher Hacon for helpful discussion.

Received by the editors February 15, 1996.

1991 Mathematics Subject Classification. Primary 14H45, 14H99; Secondary 14H50.

(C)1997 American Mathematical Society 
Lemma 1. Let

$$
f\left(x_{1}, \ldots, x_{18}\right)=\sum_{i=1}^{9} a x_{i}^{2}+\sum_{i=10}^{18} b x_{i}^{2}+\sum_{i=1}^{18} c x_{i}
$$

where $a, b \in \mathbb{N}, c \in \mathbb{Z}$, and $|a-b|=1$ or 2 . Then there exists an integer $m_{0}=$ $m_{0}(a, b)$ such that for any even integer $m \geq m_{0}, f\left(x_{1}, \ldots, x_{18}\right)=m$ has an integral solution.

Proof. 1. Suppose $(a, b)=1$ :

There exists an $n_{0} \in \mathbb{Z}$ such that for any integer $n \geq n_{0}, n=a r+b s$ for some $r, s \in \mathbb{N}$. Furthermore, every positive integer is the sum of four squares. So

$$
n=a \sum_{i=1}^{4} \bar{x}_{i}^{2}+b \sum_{i=5}^{8} \bar{x}_{i}^{2}
$$

for some $\bar{x}_{1}, \ldots, \bar{x}_{8} \in \mathbb{Z}$. Hence for any even integer $m \geq 2 n_{0}$,

$$
m=f\left(\bar{x}_{1}, \ldots, \bar{x}_{4},-\bar{x}_{1}, \ldots,-\bar{x}_{4}, 0, \bar{x}_{5}, \ldots, \bar{x}_{8},-\bar{x}_{5}, \ldots,-\bar{x}_{8}, 0\right)
$$

for some $\bar{x}_{1}, \ldots, \bar{x}_{8} \in \mathbb{Z}$.

2. Suppose $(a, b)=2$ :

(i) Suppose $m \equiv 0(\bmod 4)$ :

We write $\frac{m}{4}$ as a combination of $\frac{a}{2}$ and $\frac{b}{2}$ for $m \gg 0$. So similiarly,

$$
m=f\left(\bar{x}_{1}, \ldots, \bar{x}_{4},-\bar{x}_{1}, \ldots,-\bar{x}_{4}, 0, \bar{x}_{5}, \ldots, \bar{x}_{8},-\bar{x}_{5}, \ldots,-\bar{x}_{8}, 0\right)
$$

for some $\bar{x}_{1}, \ldots, \bar{x}_{8} \in \mathbb{Z}$.

(ii) Suppose $m \equiv 2(\bmod 4)$ :

Then we have $a+b \equiv 2(\bmod 4)$ and $m-a-b \equiv 0(\bmod 4)$. Hence for $m \gg 0$

$$
\begin{gathered}
m-a-b=f\left(\bar{x}_{1}, \ldots, \bar{x}_{4},-\bar{x}_{1}, \ldots,-\bar{x}_{4}, 0, \bar{x}_{5}, \ldots, \bar{x}_{8},-\bar{x}_{5}, \ldots,-\bar{x}_{8}, 0\right), \\
m=f\left(\bar{x}_{1}, \ldots, \bar{x}_{4},-\bar{x}_{1}, \ldots,-\bar{x}_{4}, 1, \bar{x}_{5}, \ldots, \bar{x}_{8},-\bar{x}_{5}, \ldots,-\bar{x}_{8},-1\right) .
\end{gathered}
$$

for some $\bar{x}_{1}, \ldots, \bar{x}_{8} \in \mathbb{Z}$. This proves the lemma.

Remark 1. From the above proof, $\sum_{i=1}^{4} a \bar{x}_{i}^{2} \leq m$ and $\sum_{i=5}^{8} b \bar{x}_{i}^{2} \leq m$. So $\bar{x}_{i} \leq \sqrt{m}$. We can modify Lemma 1 as:

Given $f$, for all even integer $m \gg 0, m$ can be represented by $f\left(x_{1}, \ldots, x_{18}\right)$ with $\left|x_{i}\right| \leq \sqrt{m}$ for all $i$.

\section{MAIN THEOREM}

It's enough to prove the theorem when $\operatorname{dim} X=3$. So let $X$ be a smooth projective threefold. We first construct curves $C_{1}$ and $C_{1}^{\prime} \subset X\left(C_{1}\right.$ smooth but $C_{1}^{\prime}$ singular) whose arithmetic genera differ by one. Let $H_{i}(i=1 \ldots 5)$ denote linearly equivalent very ample divisors in $X$. Consider

$$
C_{1}=\left(H_{1} \cap H_{2}\right) \cup\left(H_{3} \cap H_{4}\right), C_{1}^{\prime}=\left(H_{1} \cap H_{2}\right) \cup\left(H_{3} \cap H_{5}\right) .
$$

Choose $H_{i}$ 's properly so that $C_{1}$ is smooth but disconnected (with $H_{1} \cap H_{2}$ and $H_{3} \cap$ $H_{4}$ as its two components), and $C_{1}^{\prime}$ is connected and has a simple node at $H_{1} \cap$ $H_{2} \cap H_{3} \cap H_{5}$. Such $C_{1}$ and $C_{1}^{\prime}$ have the same intersections with divisors in $X$, and their arithmetic genera differ by one. By moving the very ample divisors, we can construct more curves, $C_{1}, \ldots, C_{18}$, such that 
1. They all are disjoint.

2. For all divisors $D \subset X, \forall 1 \leq i, j \leq 18$,

$$
C_{i} \cdot D=C_{j} \cdot D \text {. }
$$

3. $p_{a}\left(C_{i}\right)=p_{a}\left(C_{j}\right)+1, \forall 1 \leq i \leq 9,10 \leq j \leq 18$.

Fix next a very ample divisor $H$ in $X$ and a smooth surface $S \in|n H|(n \gg 0)$ containing all curves constructed above. Computing on $S$ for any of our curves $C$ :

$$
2 p_{a}(C)-2=K_{X} \cdot C+n H \cdot C+C \cdot{ }_{S} C .
$$

Let

$$
C_{i} \cdot C_{i}=-a, \text { for all } 1 \leq i \leq 9
$$

then

$$
C_{i} \cdot C_{i}=-(a+2), \text { for all } 10 \leq i \leq 18 .
$$

Since we chose $S \in|n H|$ with $n \gg 0$, we may assume that $a>0$.

Next, let $H_{S}$ denote the restriction of the very ample divisor $H$ in $X$ to $S$. Let

$$
\begin{gathered}
D_{m, x_{1}, \ldots, x_{18}} \equiv K_{S}+m H_{S}+\sum_{i=1}^{18} x_{i} C_{i}, \\
D_{m} \equiv K_{S}+m H_{S} .
\end{gathered}
$$

The plan is this: We show that for $\left|x_{i}\right|$ small compared to $m$, the linear series $\left|D_{m, x_{1}, \ldots, x_{18}}\right|$ is very ample. By using Lemma 1 , we show that by choosing suitable $x_{i}$ we can find curves in their linear series of all possible genera sufficiently close to $p_{a}\left(D_{m}\right)$. Then we let $m$ vary.

Lemma 2. For any $e>0$, there exists positive integer $m_{1}=m_{1}(e)$ such that $\left|D_{m, x_{1}, \ldots, x_{18}}\right|$ is very ample $\forall\left(m \geq m_{1},\left|x_{i}\right| \leq e \sqrt{m}\right)$. In particular, there exist smooth curves in $\left|D_{m, x_{1}, \ldots, x_{18}}\right|$.

Proof. We prove the very ampleness of adjoint linear series by Reider's Theorem [2]. We need to show that for any irreducible curve $\Gamma \subset S$,

$$
\left(D_{m, x_{1}, \ldots, x_{18}}-K_{S}\right) \cdot \Gamma \geq 3
$$

and

$$
\left(D_{m, x_{1}, \ldots, x_{18}}-K_{S}\right)^{2} \geq 10 .
$$

We can find an integer $t$ such that $\left|t H_{S}-\sum_{i=1}^{18} \epsilon_{i} C_{i}\right|$ are very ample for any $\epsilon_{i}=1$, 0 , or -1 , and hence $\left|t H_{S}-\sum_{i=1}^{18} \epsilon_{i} C_{i}\right|$ has positive intersection with any effective curve. If we pick $m$ such that $m>18 t e \sqrt{m} \geq 18 t\left|x_{i}\right|$, easy computation shows that these are indeed the case.

Proof of Main Theorem. Let

$$
\begin{gathered}
g_{m}=p_{a}\left(D_{m}\right), \\
S_{m}=\left\{p_{a}\left(D_{m, x_{1}, \ldots, x_{18}}\right) \mid m, x_{i} \in \mathbb{Z}\right\} .
\end{gathered}
$$

Note that

$$
p_{a}\left(D_{m, x_{1}, \ldots, x_{18}}\right)=g_{m}-\frac{1}{2} f\left(x_{1}, \ldots, x_{18}\right),
$$


where

$$
f\left(x_{1}, \ldots, x_{18}\right)=\sum_{i=1}^{9}(a+2) x_{i}^{2}+\sum_{i=10}^{18} a x_{i}^{2}+\sum_{i=1}^{18} m\left(H \cdot C_{i}\right) x_{i}
$$

satisfies the condition of Lemma 1.

We first show that for any integer $z \leq g_{m-1}, z$ will be in $S_{m}$. This can be done by Lemma 1 and letting $m \gg 0$ because

$$
g_{m}-z \geq g_{m}-g_{m-1}=m H_{S}^{2}+o(1) .
$$

Secondly, we want to show that for any integer $z, g_{m-2} \leq z \leq g_{m-1}, z$ is actually genus of some smooth curves if $m \gg 0$. Choose $e$ so that $e^{2} m \geq 2\left(g_{m}-g_{m-2}\right), \forall m \gg$ 0 . Hence for any integer $z, g_{m-2} \leq z \leq g_{m-1}, z=p_{a}\left(D_{m, x_{1}, \ldots, x_{18}}\right)$ for some $\left|x_{i}\right| \leq \sqrt{2\left(g_{m}-z\right)} \leq e \sqrt{m}$ [Remark 1]. By Lemma 2, such $z$ can be realized as genus of some smooth curves.

Finally, let $m$ vary. Every integer $g$ lies inside some interval $\left[g_{m-2}, g_{m-1}\right]$. This completes the proof.

\section{VARIANTS, AND EXAMPLE}

We can't expect to realize all large genera by smooth curves on a general surface. The best thing we can hope is allowing curves to have some simple nodes.

Theorem 2. Let $S$ be a smooth surface. Then there exists an integer $g_{0}=g_{0}(S)$ such that for any integer $g \geq g_{0}$, there exist nodal curves with geometric genus $=g$.

Proof. Let $H$ be a very ample divisor on $S$. Let

$$
\begin{gathered}
g_{m}=p_{a}\left(K_{S}+m H\right), \\
d_{m}=g_{m}-g_{m-1} .
\end{gathered}
$$

It is enough to show that for $m \gg 0$, and $\forall r, 1 \leq r \leq d_{m}$, there exists a nodal curve $C \in\left|K_{S}+m H\right|$ with exactly $r$ simple nodes.

Let $C_{1} \in\left|\left[\frac{m}{4}\right] H\right|$ be a smooth curve in $S$. Let $\Sigma=\left\{x_{1}, \ldots, x_{r}\right\}$ be $r$ distinct points in $C_{1}$. Now consider the blowup of $S$ along $\Sigma$

$$
f: \tilde{S}=B l_{\Sigma}(S) \rightarrow S .
$$

Let $E_{i}$ 's denote the exceptional divisors over $x_{i}$ 's. Let

$$
L_{m, r}=m f^{*} H-3\left(E_{1}+\ldots+E_{r}\right) .
$$

Then

$$
\begin{gathered}
K_{\tilde{S}}+L_{m, r} \equiv f^{*}\left(K_{S}+m H\right)-2\left(E_{1}+\ldots+E_{r}\right), \\
L_{m, r} \equiv\left(m-3\left[\frac{m}{4}\right]\right) f^{*} H+3 \tilde{C}_{1},
\end{gathered}
$$

where $\tilde{C}_{1}$ denotes the proper transformation of $C_{1}$.

Computation shows that, for $m$ large enough, $L_{m, r}$ has both self-intersection and intersection with effective curves positive enough. So $\left|K_{\tilde{S}}+L_{m, r}\right|$ is very ample by Reider's Theorem. Then we can pick a curve $\tilde{C} \in\left|K_{\tilde{S}}+L_{m, r}\right|$ such that $\tilde{C}$ is smooth and $\tilde{C}$ intersects $E_{i}$ at 2 distinct points $\forall 1 \leq i \leq r$. Hence $C:=f_{*}(\tilde{C})$ is a nodal curve in $\left|K_{S}+m H\right|$ with $r$ simple nodes at $x_{1}, \ldots, x_{r}$. This completes the proof. We left the details to the readers. 
Turning back to the higher dimensional case, we conclude with an elementary example to show that the least integer $g_{0}$ appearing in Theorem 1 can be arbitrarily large:

Example. Let $X=C_{1} \times C_{2} \times C_{3}$, where $C_{i}$ 's are smooth projective curves. There are natural projections $p_{i}$ 's to each $C_{i}$ 's. For any smooth irreducible curve $C$ in $X$, not all the projections of $C$ are points. Hence we have:

$$
g(C) \geq \min \left(g\left(C_{1}\right), g\left(C_{2}\right), g\left(C_{3}\right)\right) .
$$

Hence there is no uniform $g_{0}$ for all projective varieties of same dimension.

\section{REFERENCES}

1. H.Clemens, Curves on Generic Hypersurfaces, Ann. Sci. Ecole Norm. Sup. 19 (1986), 629636. MR 88c: 14037

2. R.Lazarsfeld, Lectures on Linear Series, preprints (1994).

Department of Mathematics, University of California at Los Angeles, Los Angeles, CALIFORNIA 90095-1555

E-mail address: jachen@math.ucla.edu 